\title{
Neurophysiological findings in patients 1 year after snake bite-induced neurotoxicity in Sri Lanka
}

\author{
D.J. Bell ${ }^{\mathrm{a}, \mathrm{b}, *}$, D. Wijegunasinghe ${ }^{\mathrm{c}}$, S. Samarakoon ${ }^{\mathrm{c}}$, H. Palipana $^{\mathrm{c}}$, S. Gunasekera ${ }^{\mathrm{d}}$, \\ H.A. de Silva ${ }^{\mathrm{b}}$, D.G. Lalloo ${ }^{\mathrm{e}}$, U.K. Ranawaka ${ }^{\mathrm{b}}$, H.J. de Silva ${ }^{\mathrm{b}}$ \\ a Tropical and Infectious Diseases Unit, Royal Liverpool University Hospital, Liverpool L7 8XP, UK \\ ${ }^{\mathrm{b}}$ Faculty of Medicine, University of Kelaniya, Ragama, Sri Lanka \\ c Department of Neurology, Kurunegala Teaching Hospital, Kurunegala, Sri Lanka \\ d Institute of Neurology, National Hospital of Sri Lanka, Colombo, Sri Lanka \\ e Clinical Group, Liverpool School of Tropical Medicine, Liverpool L3 5QA, UK
}

\section{A R T I C L E I N F O}

\section{Article history:}

Received 30 April 2009

Received in revised form

10 December 2009

Accepted 10 December 2009

Available online $\mathrm{xxx}$

\section{Keywords:}

Snake bite

Venom

Neurotoxicity

Neurophysiology

Demyelination

Electromyography

\begin{abstract}
A B S T R A C T
Snake bite causes significant morbidity and mortality in Sri Lanka. Snake venoms contain neurotoxins that block neuromuscular junction transmission. Pre-synaptic neurotoxicity most commonly causes destruction of nerve terminals with recovery by re-growth, whilst post-synaptic neurotoxicity usually involves competition at the acetylcholine receptor. The aim of this study was to investigate whether there were long-term clinical or neurophysiological changes in snake bite survivors 1 year after their envenoming. Detailed neurophysiological tests and clinical examinations were performed on 26 snake bite victims who had presented with neurotoxicity 12 months previously, and their results were compared with controls recruited from the same communities. Significant differences were observed in some nerve conduction parameters in some snake bite cases compared with controls, predominantly in those thought to be due to elapid bites, including prolongation of sensory, motor and F-wave latencies and reduction of conduction velocities. There was no evidence of any residual deficits in neuromuscular junction transmission. These results suggest a possible demyelinating type polyneuropathy. None of the cases or controls had abnormalities on clinical examination. This is one of the few studies to report possible long-term neurological damage following systemic neurotoxicity after snake bite. The clinical significance of these neurophysiological abnormalities is uncertain and further studies are required to investigate whether the abnormalities persist and to see whether clinical consequences develop.
\end{abstract}

(c) 2009 Published by Elsevier Ltd on behalf of Royal Society of Tropical Medicine and Hygiene.

\section{Introduction}

Sri Lanka has a high incidence rate of snake bite, with around 30000 bites and 100 deaths occurring annually. ${ }^{1}$ More than $97 \%$ of these deaths are due to bites from elapids [cobras (Naja naja) and common kraits (Bungarus

\footnotetext{
* Corresponding author. Tel.: +44151 706 3835; fax: +44 1517065944 .

E-mail address: belldavidj@gmail.com (D.J. Bell).
}

caeruleus)] or Russell's viper (Daboia russelii russelii). The venoms of these snakes contain powerful neurotoxins causing a progressive neuromuscular paralysis by blockade of neuromuscular transmission at the nerve terminal and neuromuscular junction. The venom of the common krait has neurotoxins that act both pre- and post-synaptically, whilst those of the Indian cobra act on the post-synaptic terminal and those of the Russell's viper are most likely to exert their effect pre-synaptically, although post-synaptic toxins have also been identified. ${ }^{2-6}$ Post-synaptic neuro- 
toxicity normally involves competition at the acetylcholine receptor, whereas pre-synaptic neurotoxicity most commonly occurs after irreversible binding and destruction of the nerve terminal, with recovery by re-growth of the nerve terminal. ${ }^{7,8}$ In addition, the venom of Russell's viper contains toxins causing coagulopathy.

The clinical manifestations of neurotoxicity following envenoming include ptosis, ophthalmoplegia and respiratory failure that may require ventilation. Most patients will make a full clinical recovery within days of the bite if supported during the acute phase after receiving antivenom (AV). The aim of this study was to investigate whether there were any long-term clinical or neurophysiological changes in snake bite survivors 1 year after their envenoming. Cases who had previously been enrolled in an AV study in 2007 after presenting with features of systemic neurotoxicity and who had been treated with AV at that time were recruited. Detailed neurophysiological testing was performed on all these cases and the results were compared with a control group.

\section{Methods}

\subsection{Participants and study location}

Cases were identified from the records of an AV study that had taken place between March 2005 and April 2008 investigating the role of different pre-medications in patients about to receive AV following envenoming. All the patients included in this study had presented to the Teaching Hospital, Kurunegala, Sri Lanka, approximately 100 km north of the capital city Colombo, in 2007, with features of neurotoxicity and all had received AV. Anticholinesterases were not administered.

Letters were sent to potential patients inviting them to attend the hospital for a neurological assessment to see how well they had recovered from their snake bite. Control data were obtained from relatives or friends who accompanied the cases to the study clinic and who were willing to participate in the study. By recruiting family members and friends it was expected that cases and controls would be similar in terms of socioeconomic status, nutritional status and geographical residence. Most snake bite victims in Sri Lanka work in rural areas where organophosphate and other agrochemicals are commonly used, and exposure to these compounds is likely to be similar in both groups.

\subsection{Clinical procedures}

On arrival at the study clinic, the study was explained in further detail and participants were asked to give written informed consent. Cases or controls were excluded if they had a history of excessive alcohol intake, leprosy or if they were receiving therapy for tuberculosis (TB) or diabetes mellitus (DM). Baseline demographic details were collected and a random blood sugar was measured. The neurological assessment included a detailed clinical questionnaire relating to possible neurological symptoms noticed by the participant. This was followed by a detailed neurological examination, which, for consistency, was performed by one of two members of the study team (DJB and UKR).
Neurophysiological evaluation was done using standard techniques and was carried out by the same investigator (DW), a consultant neurologist, who was blinded to whether the patient was a case or control. All studies were performed using a Nihon Kohden Neuropack EMG machine (model MEB 5504K; Nihon Kohden, Tokyo, Japan) in an air-conditioned room with the ambient temperature maintained approximately $22^{\circ} \mathrm{C}$. Patients were seen on one occasion only and the neurophysiological studies were not repeated at a later date. The following tests were performed.

\subsubsection{Sensory nerve conduction studies of the left ulnar nerve and left sural nerve}

The peak amplitude of the sensory action potential (SAP), latency to the peak of the SAP, and sensory conduction velocity (SCV) using onset latency were measured. Ulnar nerve (UN) sensory conduction was assessed using ring electrodes over the fifth digit to stimulate the UN orthodromically, with surface recording electrodes placed $12 \mathrm{~cm}$ proximally in the wrist. For the sural nerve, surface recording electrodes were placed over the lateral aspect of the ankle posterior to the lateral malleolus, with stimulating electrodes placed $14 \mathrm{~cm}$ proximally over the posterior foreleg. Maximal SAPs were recorded using supramaximal stimulation.

\subsubsection{Motor nerve conduction studies of the left ulnar nerve and left posterior tibial nerve}

The distal latency of the compound motor action potential (CMAP), peak amplitude of the CMAP, motor conduction velocity (MCV) and the minimum F-wave latencies were measured. The posterior tibial (PT) nerve was selected for motor studies in the lower limb to minimise the effect of possible compression neuropathy of the common peroneal nerve in this population largely involved with farming and heavy manual work. Motor nerve conduction studies were performed using surface stimulating electrodes placed over the wrist (UN) and ankle posterior to the medial malleolus (PT nerve) and recording electrodes placed over the abductor digiti minimi (ADM) and abductor hallucis (AH), respectively, after supramaximal stimulation. MCVs were recorded by stimulating at two points at a fixed distance apart. Minimum F-wave latencies were recorded as the shortest latencies of 16 supramaximal stimuli of the UN and PT nerve with recording electrodes placed over the over $\mathrm{ADM}$ and $\mathrm{AH}$, respectively.

\subsubsection{Repetitive nerve stimulation of the left ulnar nerve and left posterior tibial nerve}

The percent change in the amplitude of the motor action potential over the ADM for the UN and the AH for the PT nerve were recorded. Nerves were stimulated supramaximally at $3 \mathrm{~Hz}$ over the wrist for the UN and the ankle for the PT nerve for a total of five stimuli and the percent change in amplitude after the fourth response compared with the first response was recorded. A decrease of $>10 \%$ in amplitude after the fourth response was considered abnormal. 
Table 1

Baseline characteristics [mean (range)] of cases and controls

\begin{tabular}{llll}
\hline Characteristic & Cases $(N=26)$ & Controls $(N=22)$ & $P$-value a \\
\hline Age $($ years $)$ & $39.8(13-73)$ & $39.0(14-64)$ & 0.850 \\
Height $(\mathrm{cm})$ & $153.4(122-179)$ & $154.4(135-173)$ & 0.896 \\
Weight $(\mathrm{kg})$ & $51.9(35-75)$ & $55.2(25-74)$ & 0.379 \\
RBS $(\mathrm{mg} / \mathrm{dl})$ & $117.9(83-215.0)$ & $129.2(91-243.0)$ & 0.251 \\
Years in education & $8.7(1-12)$ & $8.1(0-12)$ & 0.598 \\
\hline
\end{tabular}

RBS: random blood sugar.

${ }^{\text {a }}$ Calculated by the $t$-test.

\subsubsection{Electromyography studies of small muscles of the hand and foot}

Standard electromyography (EMG) studies were performed using concentric needle electrodes in the left $\mathrm{ADM}$ and left $\mathrm{AH}$ to assess insertional, spontaneous and volitional activity. If abnormalities were noted in these muscles, further recordings were made from additional muscles, including the right $\mathrm{ADM}$, right $\mathrm{AH}$, genioglossus and paraspinalis.

\subsection{Statistics}

Baseline characteristics were summarised as the mean and range. Continuous variables were compared by $t$-test and categorical data by $\chi^{2}$ test. Nerve conduction results were not normally distributed and were summarised by median and interquartile range and were compared using the Mann-Whitney test with a significance level set at $P<0.05$.

\section{Results}

Between June and November 2008 letters were sent to 80 patients known to have had systemic neurotoxicity in 2007 after snake bite. Twenty-six cases responded to the invitation and were recruited to the study along with 22 controls. No participants were excluded because of excessive alcohol intake, treatment for DM or TB, or leprosy. Baseline characteristics of the two groups are compared in Table 1 . There were $16 / 26$ males (61.5\%) in the case group compared with $8 / 22(36.4 \%)$ in the control group $(P=0.147$, Fishers exact). There were no differences in any of the characteristics shown in Table 1 between cases and controls. The majority of cases and controls were farmers or spouses of farmers [18/26 (69.2\%) and 12/22 (54.5\%), respectively; $P=0.375]$. There was no difference in the average household income between the cases and controls.

In total, $8 / 26$ cases (30.8\%) and $11 / 22$ controls (50\%) complained of numbness in one or more of their limbs $(P=0.239$, Fishers exact). However, no objective abnormalities in sensation for light touch, pin-prick or joint position sense were detected in any of the participants. The remainder of the neurological examinations, including tone, power, co-ordination, reflexes and cranial nerves, were all normal.

All 26 cases had presented with features of systemic neurotoxicity, most commonly ptosis, diplopia and ophthalmoplegia. The biting species was definitively identified in only 3 of the 26 cases at the time of their presentation to hospital; in all 3 cases the snake was a Russell's viper.
An additional seven cases had features of coagulopathy, including haematuria, gum bleeding and haematemesis, suggesting that these patients were also envenomed by a Russell's viper. For the remaining 16 cases, no snake species was positively identified, but the presence of neurotoxicity in the absence of coagulopathy makes it most likely that these were elapids (cobra or kraits).

Following snake bite, the median duration of admission to hospital was 4 days (range 2-28 days). None of the cases required mechanical ventilation during their admission. The mean time from snake bite to recruitment to the study was 464 days (range 377-534 days). None of the controls gave a past history of snake bite, however four of the cases had been bitten once before prior to 2007. All 26 cases were bitten on their lower limbs, 13 (50\%) on the right leg and $13(50 \%)$ on the left leg. Two patients with previously undiagnosed DM were recruited, one into each of the groups, however they were not excluded because they were not on treatment for DM. None of the participants were on any medication and none had clinical features suggestive of leprosy.

\subsection{Nerve conduction and electromyography studies}

Results of the sensory and motor nerve conduction studies are shown in Tables 2 and 3, respectively. Comparisons are made between the results for the controls and all 26 cases together, and then between the controls and the 16 presumed elapid snake bites and the 10 presumed Russell's viper bites.

UN and sural nerve SAP peak latencies were prolonged in cases compared with controls $(P=0.029$ and 0.047 , respectively) when all the cases were considered together. However, after dividing the cases according to the presumed biting species of snake, differences in sensory nerve conduction were seen only in the UN, and not the sural nerve, after envenoming due to a presumed elapid bite; there were no differences between controls and cases following a presumed Russell's viper bite. After a presumed elapid bite, the UN peak latency was prolonged, the UN peak amplitude was increased and UN SCV was reduced compared with controls $(P=0.005,0.040$ and 0.013 , respectively).

Distal motor latencies and minimum F-wave latencies were prolonged in the UN of cases compared with controls in the motor conduction studies $(P=0.02$ and 0.022 , respectively) when all the cases were considered together. In addition, the PT nerve MCV was reduced in snake bite cases $(P=0.031)$. These differences were only seen after envenoming due to a presumed elapid; there were no differences between controls and cases thought to be due to Russell's viper bites. After a presumed elapid bite, the UN distal motor latency and minimum F-wave latencies were prolonged compared with controls $(P=0.036$ and 0.017 , respectively). There was also a strong trend towards a reduced PT nerve $\operatorname{MCV}(P=0.056)$. No other significant differences were seen in the motor nerve conduction studies between cases and controls.

For the repetitive nerve stimulation tests, only one participant, a snake bite case due to a presumed Russell's viper, fulfilled the predefined abnormality criteria with a 
Table 2

Results of sensory nerve conduction studies in controls and cases (cases are shown grouped together and divided according to presumed snake species involved)

\begin{tabular}{|c|c|c|c|c|c|c|c|}
\hline & $\begin{array}{l}\text { Controls } \\
(N=22) \\
{[\text { median (IQR)] }}\end{array}$ & $\begin{array}{l}\text { All cases } \\
(N=26) \\
{[\text { median }(\mathrm{IQR})]}\end{array}$ & $\begin{array}{l}P \text {-value } \\
\text { (controls vs. all cases) }^{\mathrm{a}}\end{array}$ & $\begin{array}{l}\text { Elapids } \\
(N=16) \\
\text { [median (IQR)] }\end{array}$ & $\begin{array}{l}P \text {-value } \\
\text { (controls vs. elapids) }^{\mathrm{a}}\end{array}$ & $\begin{array}{l}\text { Russell's viper } \\
(N=10) \\
{[\text { median (IQR)] }}\end{array}$ & $\begin{array}{l}P \text {-value } \\
\text { (controls vs. Russell's viper) }^{\text {a }}\end{array}$ \\
\hline \multicolumn{8}{|l|}{ Ulnar nerve } \\
\hline Latency to SAP peak & $2.70(2.50-2.85)$ & $2.84(2.66-3.16)$ & 0.029 & $3.05(2.72-3.17)$ & 0.005 & $2.71(2.58-2.95)$ & 0.633 \\
\hline Peak amplitude of SAP & $6.30(3.75-13.15)$ & $9.65(7.27-14.42)$ & 0.104 & $10.5(8.25-14.3)$ & 0.040 & $7.2(4.02-14.6)$ & 0.724 \\
\hline SCV & $54.10(50.20-58.30)$ & $51.70(46.20-55.60)$ & 0.161 & $49.4(46.2-53.47)$ & 0.013 & $55.6(50.22-59.2)$ & 0.546 \\
\hline \multicolumn{8}{|l|}{ Sural nerve } \\
\hline Latency to SAP peak & $3.39(3.11-3.88)$ & $3.71(3.39-3.87)$ & 0.047 & $3.72(3.34-3.88)$ & 0.155 & $3.68(3.61-3.86)$ & 0.053 \\
\hline Peak amplitude of SAP & $18.15(14.20-22.92)$ & $18.45(8.47-25.77)$ & 0.959 & $19.1(12.0-27.8)$ & 0.630 & $12.5(6.8-23.65)$ & 0.433 \\
\hline SCV & $47.05(42.02-49.37)$ & $46.10(43.70-49.65)$ & 0.949 & $47.6(43.5-50.7)$ & 0.656 & $46.1(43.95-46.7)$ & 0.433 \\
\hline
\end{tabular}

IQR: interquartile range; SAP: sensory action potential; SCV: sensory conduction velocity

a Calculated by the Mann-Whitney test.

Table 3

Results of motor nerve conduction studies in controls and cases (cases are shown grouped together and divided according to the presumed snake species involved)

\begin{tabular}{|c|c|c|c|c|c|c|c|}
\hline & $\begin{array}{l}\text { Controls } \\
(N=22) \\
{[\text { median (IQR)] }}\end{array}$ & $\begin{array}{l}\text { All cases } \\
(N=26) \\
{[\text { median }(\mathrm{IQR})]}\end{array}$ & $\begin{array}{l}P \text {-value } \\
\text { (controls vs. all cases) }^{\mathrm{a}}\end{array}$ & $\begin{array}{l}\text { Elapids } \\
(N=16) \\
\text { [median (IQR)] }\end{array}$ & $\begin{array}{l}P \text {-value } \\
\text { (controls vs. elapids) }^{a}\end{array}$ & $\begin{array}{l}\text { Russell's viper } \\
(N=10) \\
\text { [median (IQR)] }\end{array}$ & $\begin{array}{l}P \text {-value } \\
\text { (controls vs. Russell's viper) a }^{\text {a }}\end{array}$ \\
\hline \multicolumn{8}{|l|}{ Ulnar nerve } \\
\hline Distal motor latency & $2.10(1.92-2.33)$ & $2.26(2.12-2.65)$ & 0.020 & $2.34(2.12-2.7)$ & 0.036 & $2.24(2.04-2.56)$ & 0.092 \\
\hline CMAP amplitude & $9.91(9.12-10.72)$ & $9.88(8.52-10.82)$ & 0.852 & $10.15(9.20-10.87)$ & 0.759 & $9.51(7.39-10.9)$ & 0.434 \\
\hline MCV & $58.70(55.27-63.80)$ & $57.65(54.23-60.37)$ & 0.222 & $55.75(52.5-59.6)$ & 0.064 & $60.0(55.65-61.0)$ & 0.889 \\
\hline Minimum onset F-wave latency & $24.70(23.40-27.75)$ & $27.15(25.55-28.35)$ & 0.022 & $27.25(25.62-29.75)$ & 0.017 & $27.15(24.5-28.07)$ & 0.219 \\
\hline RNS \% decrease & $-1.60(-2.30$ to 0.22$)$ & $-1.10(-2.10$ to 1.1$)$ & 0.517 & $-1.8(-4.5$ to 0.55$)$ & 0.827 & $-0.4(-1.57$ to 1.45$)$ & 0.163 \\
\hline \multicolumn{8}{|l|}{ Post tibial nerve } \\
\hline Distal motor latency & $3.70(3.29-4.09)$ & $3.76(3.48-3.98)$ & 0.534 & $3.76(3.45-4.14)$ & 0.569 & $3.76(3.48-3.98)$ & 0.675 \\
\hline CMAP amplitude & $9.55(7.54-10.60)$ & $9.15(7.51-10.70)$ & 0.942 & $9.41(7.58-10.7)$ & 0.827 & $9.15(7.37-10.4)$ & 0.889 \\
\hline MCV & $50.05(47.27-56.10)$ & $45.90(43.07-52.20)$ & 0.031 & $46.35(42.4-52.5)$ & 0.056 & $44.95(43.07-51.45)$ & 0.109 \\
\hline Minimum onset F-wave latency & $45.85(42.87-49.00)$ & $47.90(44.35-51.15)$ & 0.153 & $48.8(45.6-52.5)$ & 0.105 & $47.75(42.95-50.22)$ & 0.589 \\
\hline RNS \% decrease & $-0.80(-1.85$ to 0.72$)$ & 0.55 (-3.00 to 2.72$)$ & 0.398 & $1.5(-3.22$ to 3.17$)$ & 0.327 & $0(-3.0$ to 1.8$)$ & 0.734 \\
\hline
\end{tabular}

IQR: interquartile range; CMAP: compound motor action potential; MCV: motor conduction velocity; RNS: repetitive nerve stimulation.

a Calculated by the Mann-Whitney test. 
decrease of $14 \%$ in the PT nerve amplitude. The remaining participants had decreases of $<10 \%$ in amplitude after the fourth response. The EMG studies were normal for $19 / 26$ cases (73.1\%) and 16/22 controls (72.7\%). EMG for the remaining cases and controls showed features suggestive of denervation with high firing and polyphasia in the muscles tested. These EMG changes were bilateral for 11 of the 13 participants ( 5 cases and 6 controls). For five participants (two cases and three controls), denervation was observed in muscles both in the upper and lower limbs, for four participants (three cases and one control) the upper limbs only, and for four participants (two cases and two controls) the lower limb only. EMG abnormalities were seen in patients both after Russell's viper and elapid bites.

\section{Discussion}

This is one of the few studies to investigate possible long-term neurological damage following systemic neurotoxicity after snake bite. There are no national-level reference values for nerve conduction study (NCS) parameters for Sri Lankan patients and, in any event, normal parameter values vary from laboratory to laboratory and values applicable to one setting may be unsuitable in another. We have therefore compared NCS results from cases and controls recruited from the same communities whose exposure to potential environmental hazards including pesticides and heavy metals would have been similar. The ages and heights of the cases and controls as well as the male/female sex ratio and random blood sugar ranges were similar. Most of the cases and controls were paddy farmers or their spouses, and family incomes and educational status were also similar. No patient or control was on therapy for TB (isoniazid) or DM, had leprosy or had excess alcohol intake (recognised causes of neuropathy), although one case and one control were found to have raised blood sugars.

One year after snake bite, we observed significant differences in some of the NCS parameters of snake bite cases compared with controls. These include prolongation of sensory, motor and F-wave latencies as well as reduction of conduction velocities. The changes were more marked in the upper limbs than the lower limbs and mostly involved the UN. This would suggest that if these changes were related to the envenoming, the effect is systemic rather than local neurological damage at the site of the snake bite, as all cases in the study were bitten on the lower limb. We have found no evidence from the repetitive nerve stimulation tests of any residual deficits in neuromuscular junction transmission. Taken together, the results suggest a non-length-dependent demyelinating type polyneuropathy. These nerve conduction abnormalities are not typical of a toxin-medicated neuropathy, which is usually associated with axonal damage.

There are limitations to the study. Neurophysiological tests were performed on only one occasion and we cannot comment on the reproducibility of the results presented here. The biting species was only positively identified in $3 / 26$ cases, as is typical in Sri Lanka. In the larger AV study from which patients for this study were recruited (4000 patients), the species of snake was identified in $<25 \%$ of patients. In the absence of definitive immunodiagnosis, cases were divided into presumed Russell's viper on the basis of snake identification or coagulopathy, or presumed elapid species (cobra or krait). Abnormalities in nerve conduction were only observed after snake bite by a presumed elapid.

In contrast to nerve conduction, EMG abnormalities were noted both in cases and controls; 7/26 cases and $6 / 22$ controls had denervation changes on EMG. These may be due to the effects of neuropathy, unrecognised anterior horn cell degeneration and/or co-existent radiculopathies. Despite these unexplained abnormalities in the general population, significant differences in nerve conduction were still observed between snake bite cases and controls overall and in particular between presumed elapid snake bite cases and controls, making it likely that these differences are due to the effect of snake bite.

The number of electrophysiological studies in snake bite is surprisingly small. The most common finding has been decremental responses to repeated muscle stimulation in patients envenomed by species that cause pre-synaptic neurotoxicity, including the common krait in Sri Lanka. ${ }^{9-11}$ Abnormalities found in acute stages returned to normal after several days. There have been three previous studies of krait envenoming in Sri Lanka, all done in the acute phase. One showed no abnormalities of nerve conduction, ${ }^{9}$ one demonstrated prolongation of distal latency of the median nerve in 3 of 12 patients associated with reduced median CMAP amplitudes that subsequently returned to normal, ${ }^{10}$ and one demonstrated defects in nerve conduction in approximately $20 \%$ of patients that lasted between 2 weeks and 6 months. ${ }^{12}$ Electrophysiological abnormalities persisting as long as a year have never been reported. In the latter study, abnormalities also occurred both in the upper and lower limbs, as in our study, suggesting that this is a systemic rather than a local phenomenon.

The cause of these abnormalities in nerve conduction studies remains uncertain. The venom of kraits contains both $\alpha$ - and $\beta$-bungarotoxins; the lack of responsiveness to $A V$ seen in most krait bites in Sri Lanka suggest that $\beta$-bungarotoxins acting pre-synaptically predominate, causing neurotoxicity by damage to the neuromuscular junctions. ${ }^{7}$ Recovery occurs by nerve re-growth, which could potentially lead to conduction abnormalities. ${ }^{7,8}$ In contrast, envenoming by the Asian cobra ( $N$. naja) causes predominantly post-synaptic envenoming, which does not usually involve nerve damage. ${ }^{3}$ This might suggest that the nerve conduction abnormalities are more likely to be due to krait venom than cobra venom. We were unable to distinguish clinically between bites by these two species, although previous experience suggests that krait bites are the commonest elapid bites in this region. ${ }^{12}$ It is also possible that unidentified components of the venom could be causing these abnormalities. We did not identify nerve conduction problems in those bitten by presumed Russell's viper. The mechanism of neurotoxicity in Sri Lankan Russell's viper is less clear and there is geographic variation between Russell's viper subspecies: phospholipase A2 toxins, likely to act pre-synaptically, clearly play a part, but post-synaptic toxins have also been identified. ${ }^{6,13}$ 
Despite electrophysiological changes, there was no objective evidence of clinical abnormalities on neurological examination of any of the cases or controls in this study, including those with nerve conduction abnormalities and those with subjective complaints of 'numbness' in their hands or feet. The clinical significance of these neurophysiological abnormalities in snake bite cases therefore remains uncertain. It is possible that we are seeing either the recovery phase of an early insult to the peripheral nerves or the initial phase of a more chronic process. As this study only examined nerve conduction at one point in time, this issue cannot be resolved. However, our findings suggest a potential for long-term neurological sequelae following snake bite. Further larger prospective longitudinal studies are needed to confirm these findings, to investigate whether NCS abnormalities persist and to establish the potential future clinical consequences of these nerve conduction abnormalities.

Authors' contributions: DJB, DW, HAdS, DGL, UKR and HJdS developed the study idea and design; DJB, DW, SS and HP conducted the study; DW, SG and UKR analysed the neurophysiological results; DJB, SG, DGL, UKR and HJdS analysed the data and drafted the manuscript. All authors reviewed the manuscript and approved the final version. HJdS and DGL are guarantors of the paper.

Acknowledgements: The authors wish to thank the nurses and other staff at Kurunegala Teaching Hospital (Kurunegala, Sri Lanka) who assisted in the running of this study. They also thank the Hospital Director for allowing the study to take place. Finally, they wish to acknowledge all the participants (cases and controls) for agreeing to take part in the study.

Funding: None.

Conflicts of interest: None declared.
Ethical approval: This study received ethical approval from the Ethical Review Committee of the University of Kelaniya, Faculty of Medicine, Ragama, Sri Lanka (reference number P12/03/2008).

\section{References}

1. Kasturiratne A, Wickremasinghe AR, de Silva N, Gunawardena NK, Pathmeswaran A, Premaratna R, et al. The global burden of snakebite: a literature analysis and modelling based on regional estimates of envenoming and deaths. PLoS Med 2008;5:e218.

2. Abe T, Alema S, Miledi R. Isolation and characterization of presynaptically acting neurotoxins from the venom of Bungarus snakes. Eur J Biochem 1977;80:1-12.

3. Charles AK, Gangal SV, Deshpande SS, Joshi AP. Effects on muscle of a toxin from Indian cobra (Naja naja naja) venom. Toxicon 1982;20:1019-35.

4. Nirthanan S, Gwee MC. Three-finger $\alpha$-neurotoxins and the nicotinic acetylcholine receptor, forty years on. J Pharmacol Sci 2004;94:1-17.

5. Phillips RE, Theakston RD, Warrell DA, Galigedara Y, Abeysekera DT, Dissanayaka P, et al. Paralysis, rhabdomyolysis and haemolysis caused by bites of Russell's viper (Vipera russelli pulchella) in Sri Lanka: failure of Indian (Haffkine) antivenom. Q J Med 1988;68:691-715.

6. Shelke RR, Sathish S, Gowda TV. Isolation and characterization of a novel postsynaptic/cytotoxic neurotoxin from Daboia russelli russelli venom. J Pept Res 2002;59:257-63.

7. Prasarnpun S, Walsh J, Awad SS, Harris JB. Envenoming bites by kraits: the biological basis of treatment-resistant neuromuscular paralysis. Brain 2005;128:2987-96.

8. Pungercar J, Krizaj I. Understanding the molecular mechanism underlying the presynaptic toxicity of secreted phospholipases A2. Toxicon 2007;50:871-92.

9. Sanmuganathan PS. Myasthenic syndrome of snake envenomation: a clinical and neurophysiological study. Postgrad Med J 1998;74:596-9.

10. Singh G, Pannu HS, Chawla PS, Malhotra S. Neuromuscular transmis sion failure due to common krait (Bungarus caeruleus) envenomation. Muscle Nerve 1999;22:1637-43.

11. Trevett AJ, Lalloo DG, Nwokolo NC, Naraqi S, Kevau IH, Theakston RD, et al. Electrophysiological findings in patients envenomed following the bite of a Papuan taipan (Oxyuranus scutellatus canni). Trans $R$ Soc Trop Med Hyg 1995;89:415-7.

12. Kularatne SA. Common krait (Bungarus caeruleus) bite in Anuradhapura, Sri Lanka: a prospective clinical study, 1996-98. Postgrad Med J 2002;78:276-80.

13. Gopalan G, Thwin MM, Gopalakrishnakone P, Swaminathan K. Structural and pharmacological comparison of daboiatoxin from Daboia russelli siamensis with viperotoxin $\mathrm{F}$ and vipoxin from other vipers. Acta Crystallogr D Biol Crystallogr 2007;63:722-9. 